\title{
Pink Lotus Essential Oil and Alleviates on Free Fatty Acid Induced Steatosis in HepG2 Cells via PI3K/Akt and NF- $\kappa$ B Pathways
}

\author{
Runzhou Sun ${ }^{1}$, Ruixin Xiao ${ }^{1}$, Pengfei Lv ${ }^{1}$, Feifei Guo ${ }^{2}$, Yanling Gong ${ }^{1 *}$, and Meixing Yan ${ }^{3 *}$ \\ ${ }^{1}$ Department of Pharmacy, College of Chemical Engineering, Qingdao University of Science and Technology, Qingdao, Shandong, CHINA \\ ${ }^{2}$ Department of Physiology and Pathophysiology, School of Basic Medicine, Qingdao University, Qingdao, CHINA \\ ${ }^{3}$ Qingdao Women and Children's Hospital, Qingdao, CHINA
}

\begin{abstract}
Pink lotus essential oil (PLEO) is the volatile components extracted from lotus flowers and there are few relevant research. The purpose of this study was to observe the effect of PLEO on NAFLD in vitro model and its possible mechanism. The ingredients of PLEO were determined by gas chromatography-mass spectrometry (GS-MS) and its lipid-lowering and hepatoprotective activities were investigated. HepG2 cells were treated with free fatty acid (FFA) to establish a cell model of NAFLD. Cell viability was evaluated by 3-(4,5-dimethyl thiazol-2-yl)-2,5-diphenyl tetrazolium bromide (MTT) method. Total cholesterol (TC), triglyceride (TG), tumor necrosis factor- $\alpha$ (TNF- $\alpha$ ), interleukin-1 $\beta$ (IL-1 $\beta$ ), and interleukin-6 (IL-6) were determined by Enzyme-Linked Immune Sorbent Assay (ELISA). Oil red O staining was performed to observe the lipid accumulation in the HepG2 cells. Lipid metabolism enzymes including fatty acid synthase (FAS), acetyl-coA carboxylase (ACC), stearoyl-CoA desaturase 1 (SCD-1), and carnitine palmitoyltransferase-1 (CPT-1), insulin signaling pathways including phosphatidylinositol 3 kinase (PI3K) and protein kinase B Akt, inflammatory signaling pathways such as nuclear factor kappa-B (NF-KB), were determined by Western blotting. There were 46 components determined in PLEO with many terpenoids compounds. PLEO decreased TC and TG contents in the FFA-treated HepG2 cells. Furthermore, PLEO inhibited TNF- $\alpha$, IL-6 and IL-1 $\beta$ excretion, decreased NF-kB, FAS, ACC and SCD-1 while increased phosphorylation of NF-KB, PI3K, Akt, and CPT-1 expression. It is the first time to reveal that PLEO alleviates FFA-induced steatosis in HepG2 cells by regulating lipid metabolism, inhibiting inflammatory response, and improving insulin sensitivity.
\end{abstract}

Key words: pink lotus essential oil, PLEO, NAFLD, HepG2 cells, PI3K/Akt, NF-кB

\section{Introduction}

The prevalence of non-alcoholic fatty liver disease (NAFLD) has ballooned significantly in recent years with the global epidemic of obesity. The disease begins with liver steatosis and may develop into steatohepatitis with liver inflammation ${ }^{1)}$. At present, multiple-hit theory is well recognized as the pathogenesis of NAFLD ${ }^{2}$. Multiple hits, including insulin resistance, nutritional factors, hormones secreted from the adipose tissue, gut microbiota and genetic and epigenetic factors, act together to induce NAFLD in genetically predisposed subjects. However, there are no specific and effective drugs to treat NAFLD. Several antioxidants, insulin sensitizers, and lipid-lowering drugs have been applied in clinical trials to treat NAFLD/non-al- coholic steatosis $(\mathrm{NASH})^{3,4}$. It is expected to achieve satisfied therapeutic effect. Treatment of HepG2 cells with free fatty acid (FFA) is a commonly used cell model for NAFLD, which seems to reproduce the major characters of NAFLD in humans ${ }^{5}$. Excessive FFA induces cellular steatosis and toxicity, insulin resistance, oxidative stress, and inflammation, which is consistent with in vivo observations of NAFLD.

Lotus, a member of the Nelumbonaceae family, is a widely cultivated perennial aquatic plant in Asia. Its various parts, including roots, leaves, and flowers, have been used as food and herbal medicine for a long history ${ }^{6,7)}$. Studies have shown that flavonoids in lotus leaf have an obvious protective effect on liver injury induced by carbon tetra-

\footnotetext{
*Correspondence to: Yanling Gong, Department of Pharmacy, College of Chemical Engineering, Qingdao University of Science and Technology, Qingdao, Shandong, CHINA. Meixing Yan, Qingdao Women and Children's Hospital, Qingdao, CHINA E-mail: hanyu_ma@126.com (YG), meixing@163.com (MY) Accepted September 14, 2021 (received for review July 12, 2021) Journal of Oleo Science ISSN 1345-8957 print / ISSN 1347-3352 online http://www.jstage.jst.go.jp/browse/jos/ http://mc.manusriptcentral.com/jjocs

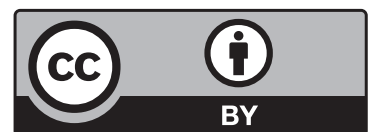


chloride $\left(\mathrm{CCl}_{4}\right)$ in mice ${ }^{8)}$. Nonedible lotus pods is a source of natural antioxidants and anticancer agents ${ }^{9)}$. Lotus seedpod extracts have anti-inflammatory effects on lipopolysaccharide (LPS) induced liver inflammation ${ }^{10)}$. However, there has been little research on lotus flowers. Pink lotus essential oil (PLEO) is a volatile component extracted from lotus flowers that gives it a special aroma. PLEO might have a variety of activities due to its complex composition. Therefore, it is necessary to explore the composition and its pharmacological activity for further development.

In the present study, we explored the protective effects of PLEO on lipid accumulation and inflammation induced by FFA in HepG2 cells. Furthermore, the potential molecular mechanisms involving in the inflammation and insulin signal pathway were discussed as well.

\section{Materials and Methods}

\subsection{Chemicals and reagents}

PLEO was obtained from Healzone Co. Ltd. (Qingdao, China). Fetal bovine serum(FBS), Dulbecco's modified Eagle's medium (DMEM), penicillin-streptomycin solution were purchased from Gibco (Carlsbad, CA, USA). The HepG2 cells were purchased from Fuheng Biology, Co. Ltd. (Shanghai, China). Total cholesterol(TC), triglyceride (TG), tumor necrosis factor $\alpha(\mathrm{TNF}-\alpha)$, interleukin-1 $\beta$ (IL-1 $\beta$ ) and interleukin-6 (IL-6) ELISA kits were purchased from Nanjing Jiancheng Bioengineering Institute(Nanjing, China). Rabbit antibodies for fatty acid synthase (FAS) (ab133619), acetyl-coA carboxylase (ACC) (ab45174), stearoyl-CoA desaturase-1 (SCD-1) (ab236868), carnitine palmitoyltransferase-1 (CPT-1) (ab234111), phosphatidylinositol 3 kinase (PI3K) (ab131067), phosphorylated PI3K (p-PI3K) (ab278545), protein kinase B (Akt) (ab8805), phosphorylated Akt (p-Akt) (ab38449), nuclear factor kappa-B p65 (NF-кB p65) (ab16502), phosphorylated nuclear factor kappa-B p65 (p-NF-кB p65) (ab183559), and glyceraldehyde-3-phosphate dehydrogenase (GAPDH) (ab245355) were obtained from Abcam (Cambridge, UK). RIPA lysis buffer, BCA kit, sodium dodecyl sulfate-polyacrylamide gel electrophoresis (SDS-PAGE), polyvinylidene difluoride(PVDF) membranes, ECL substrate kit, 3- (4,5-dimethyl thiazol-2-yl)-2,5-diphenyl tetrazolium bromide (MTT), phosphate buffer saline (PBS) powder, dimethyl sulfoxide(DMSO), Oil red O were purchased from Beijing Bioss Biotechnology Co. Ltd. (Beijing, China). Palmitate and oleate were obtained from Shanghai Aladdin Bio-Chem (Shanghai, China).

\subsection{Gas chromatography-mass spectrometry}

Coupled gas chromatography-mass spectrometry (GCMS) analysis was performed on a Shimadzu system/GC
2010 coupled to a self-gun Shimadzu/AOC-5000 and mass detector (Shimadzu MS 2010 Plus). The column used in the present study was HP-5, and the carrier gas was high purity nitrogen. The injection port temperature was $280^{\circ} \mathrm{C}$. The programmed warming process of the column was as follows: initial temperature was $50^{\circ} \mathrm{C}$ maintaining for $2 \mathrm{~min}$, and then programmed warming at $10^{\circ} \mathrm{C}$ per minute until $280^{\circ} \mathrm{C}$ for $10 \mathrm{~min}$. The column pressure was $10 \mathrm{psi}$, the shunt injection ratio was 20:1, and the injection volume was $1 \mu \mathrm{L}$. Mass spectrum conditions were as follows: the ion source was electron bombardment ion, the electron energy was $70 \mathrm{eV}$, the interface temperature was $250^{\circ} \mathrm{C}$, the mass scanning range was $m / z 50-600$, and the scanning interval was $1.0 \mathrm{~s}$.

\subsection{Cell culture and drug treatments}

Human hepatic carcinoma cell line HepG2 was cultured in DMEM medium supplemented with 10\% FBS and 1\% penicillin-streptomycin solution. The culture was maintained at $37^{\circ} \mathrm{C}$ in a humidified $5 \% \mathrm{CO}_{2}$ incubator. When the cells reached $50-70 \%$ confluence, the MTT assay and the lipid-lowering experiment were conducted. PLEO was dissolved in DMEM medium containing 0.1\% DMSO at a concentration of $0.01,0.1,1 \mu \mathrm{g} / \mathrm{mL}$, respectively. In the lipidlowering experiment, HepG2 cells were treated with FFA mixture composed of oleate acid (OA) and palmitate acid (PA) (2:1) for $48 \mathrm{~h}$ to induce the hepatic steatosis model in vitro. After that, the experiment group was added $100 \mu \mathrm{L}$ different concentration of PLEO. The control group and FFA group were added the same volume of DMEM after treated without or with FFA, respectively. Subsequently, the lipid and cytokines in the cell culture supernatant, lipid accumulation, the expression of lipid metabolism associated enzyme and PI3K/Akt and NF-кB p65 were determined by ELISA, oil red $\mathrm{O}$ staining and Western blotting analysis, respectively.

\subsection{MTT assay}

Cell viability was measured using the MTT assay. The cells $\left(2 \times 10^{4}\right.$ cells/well) were seeded in a 96 -well cell culture dish, and then treated with different concentrations of $100 \mu \mathrm{L} \operatorname{PLEO}(0.01,0.1,1 \mu \mathrm{g} / \mathrm{mL})$ for $48 \mathrm{~h}$. The control group was treated with the same volume of DMEM containing $0.1 \%$ DMSO. Every group was set five duplicated wells. After incubation, the medium was discarded, and the cells were treated with $100 \mu \mathrm{L}$ MTT solution (1 mg/ $\mathrm{mL})$ for $1 \mathrm{~h}$. The solution was then removed, and DMSO was added to dissolve the insoluble formazan crystals. The absorbance at $570 \mathrm{~nm}$ was measured using a full-wavelength Microplate Reader(Infinite m plex, Tecan, Switzerland). The cell viability was calculated as the following:

$$
\text { cell viability }(\%)=\frac{O D_{\text {sample }}}{O D_{\text {control }}} \times 100 \%
$$




\subsection{ELISA for quantification of lipids and cytokines}

After treatment, the HepG2 cells $\left(2 \times 10^{5}\right.$ cells per well in a 24-well plate) supernatants were centrifuged at 10,000 $\times$ $g$ at $4^{\circ} \mathrm{C}$ for $10 \mathrm{~min}$ to remove debris. The contents of TC, TG, TNF- $\alpha$, IL- 6 , and IL- $1 \beta$ in the supernatants were detected with ELISA kits according to the manufacturer's instructions.

\subsection{Oil Red O staining}

The treated HepG2 cells were washed with PBS and fixed in $4 \%$ paraformaldehyde for $30 \mathrm{~min}$. After washed with distilled water, the cells were added $200 \mu \mathrm{L} 5 \%$ oil red $\mathrm{O}$ and stained at room temperature for $30 \mathrm{~min}$. Then the cells were washed with tap water and dyed with hematoxylin. The staining was observed with a microscope(BX53, Olympus, Japan) and quantified with Image J software (v1.44, Bethesda, Rockville, MD, USA).

\subsection{Western botting analysis}

The HepG2 cells $\left(1 \times 10^{6}\right.$ cells per well in a 6 -well plate) were collected and the total protein was extracted using a RIPA buffer containing protease inhibitors or phosphatase inhibitors. Bicinchoninic acid (BCA) protein assay was used to determine the protein concentrations, and $30 \mu \mathrm{g}$ of protein was loaded per sample. The total protein was separated by SDS-PAGE (10\% separating gel-5\% concentrating gel) and transferred to PVDF membranes. The membranes were blocked with $5 \%$ nonfat skim milk for $2 \mathrm{~h}$ at room temperature. After washed with TBST for 3 times, the membranes were incubated with specific antibodies for

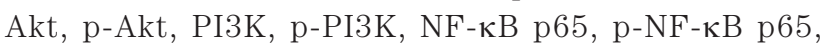
GAPDH, FAS, SCD-1, CPT-1, ACC-1 $(1: 1,000)$ for $2 \mathrm{~h}$. The membranes were then incubated with goat anti-rabbit HRP secondary antibodies $(1: 5,000)$ for $1.5 \mathrm{~h}$. The chemiluminescence detection was performed using an ECL reagent (Thermo Scientific, Massachusetts, America) and bands were developed with a gel imager(TANON-4600 Chemiluminescence Imager, Shanghai Tianneng Technology Co., Ltd. Shanghai, China). Specific bands were detected, analyzed, and quantified by Image J Software(v1.44, Bethesda, Rockville, MD, USA).

\subsection{Statistical analysis}

Statistical analysis was performed using SPSS 23.0. Data were expressed as mean \pm standard deviation $(\mathrm{SD})$. The one-way ANOVA followed with multiple comparison was used to determine significant differences among groups. Values of $\mathrm{P}$ less than 0.05 were considered to be significant.

\section{Results}

\subsection{GC-MS analysis of PLEO}

There were 46 components in the PLEO identified by
GC-MS, representing $99.85 \%$ of the total (Table 1, Fig. 1). As a complex mixture, PLEO were mainly composed of 4 groups of ingredients: aliphatic aldehydes (26.57\%), terpenoids $(25.64 \%)$, lipids $(14.89 \%)$, and aromatic hydrocarbons (14.23\%) . 2-(phenylmethylene)-Octanal (14.85\%) is the most abundant aliphatic compound detected. The identified terpenoids mainly included isolongifolol(5.83\%), L-alpha-terpineol (4.56\%), citronellol (3.63\%), geraniol $(2.64 \%)$, linalool $(2.45 \%)$, etc. It was deduced that the possible biological activities of PLEO may be attributed to terpenoids.

\subsection{Effects of PLEO on cell viability of HepG2 cells}

The effects of PLEO treatment on HepG2 cell viability were examined and shown in Fig. 2. When the cell viability is above $80 \%$, the drug can be thought to have no significant cytotoxicity on cell growth. As expected, cell viability after treated with PLEO maintained at above $80 \%$. Otherwise, it is amazing that the cell viability increased with the increase of PLEO concentration.

\subsection{Effects of PLEO on FFA-induced lipid accumulation in HepG2 cell}

The effects of PLEO treatment on lipid content in the FFA pre-treated HepG2 cells were examined and shown in Fig. 3. The results indicated that FFA pretreatment dramatically increased the intracellular TC and TG contents compared with the control(Fig. 3, $p<0.01$ ). Meanwhile, PLEO treatment dose-dependently decreased TC and TG contents in the HepG2 cells compared with the FFA group (Fig. 3, $p<0.05$ or 0.01 ). PLEO exhibited the strongest inhibition effect at a concentration of $1 \mu \mathrm{g} / \mathrm{mL}$ (Fig. 3) .

To further determine whether PLEO decreased the hepatocyte lipid accumulation in FFA-induced steatosis of HepG2 cells, Oil Red O staining were performed and quantified. There were multiple of red lipid droplets in the FFA treated HepG2 cells compared with the control group (Figs. $4 \mathrm{~A}$ and $4 \mathrm{~B}, p<0.01$ ), indicating an accumulation of lipid in the cells. Compared with the FFA group, the PLEO-treated HepG2 cells showed a decrease in cellular lipid accumulation(Figs. 4B-4E, $p<0.05$ or 0.01 ). Combined with the above results, it is revealed that PLEO ameliorated the hepatic lipid droplets induced by FFA treatment.

\subsection{Effects of PLEO on FFA-induced expression of in- flammatory mediators in HepG2 cells}

The effect of PLEO on inflammatory cytokines secreted by HepG2 cells was also observed. The results showed that TNF- $\alpha$, IL- 6 , and IL-1 $\beta$ in the HepG2 significantly increased after FFA treatment compared with the control group (Figs. 5A-5C, $p<0.01$ ). As expected, FFA induced an inflammation in addition to a lipid accumulation. Moreover, PLEO inhibited TNF- $\alpha$, IL- 6 , and IL- $1 \beta$ secreted by FFA treated HepG2 cells in different degrees (Figs. 5A-5C, $p<$ 
Table 1 Analysis results of PLEO by GC-MS.

\begin{tabular}{|c|c|c|c|c|c|}
\hline No. & Compounds & Rt, min & $\begin{array}{l}\text { Chemical } \\
\text { formula }\end{array}$ & $\mathrm{MW}, \mathrm{g} / \mathrm{moL}$ & Content, $\%$ \\
\hline 1 & D-Limonene & 6.533 & $\mathrm{C}_{10} \mathrm{H}_{16}$ & 136.234 & 0.087 \\
\hline 2 & Benzyl alcohol & 6.723 & $\mathrm{C}_{7} \mathrm{H}_{8} \mathrm{O}$ & 108.138 & 0.069 \\
\hline 3 & Benzoic acid, methyl ester & 7.684 & $\mathrm{C}_{8} \mathrm{H}_{8} \mathrm{O}_{2}$ & 136.148 & 0.084 \\
\hline 4 & Linalool & 7.759 & $\mathrm{C}_{10} \mathrm{H}_{18} \mathrm{O}$ & 154.249 & 2.445 \\
\hline 5 & Phenylethyl alcohol & 8.025 & $\mathrm{C}_{8} \mathrm{H}_{10} \mathrm{O}$ & 122.164 & 5.872 \\
\hline 6 & $(1 \mathrm{R}, 2 \mathrm{R}, 4 \mathrm{R})-2,7,7$-Trimethylbicyclo[2.2.1] heptan-2-ol & 8.535 & $\mathrm{C}_{10} \mathrm{H}_{18} \mathrm{O}$ & 154.249 & 0.183 \\
\hline 7 & Citronellal & 8.632 & $\mathrm{C}_{10} \mathrm{H}_{18} \mathrm{O}$ & 154.249 & 0.084 \\
\hline 8 & Acetic acid, phenylmethyl ester & 8.871 & $\mathrm{C}_{9} \mathrm{H}_{10} \mathrm{O}_{2}$ & 150.174 & 10.374 \\
\hline 9 & endo-Borneol & 8.933 & $\mathrm{C}_{10} \mathrm{H}_{18} \mathrm{O}$ & 154.249 & 0.161 \\
\hline 10 & L- $\alpha$-Terpineol & 9.296 & $\mathrm{C}_{10} \mathrm{H}_{18} \mathrm{O}$ & 154.249 & 4.560 \\
\hline 11 & Cyclohexanol, 1-methyl-4-(1-methylethylidene)- & 9.389 & $\mathrm{C}_{10} \mathrm{H}_{18} \mathrm{O}$ & 154.249 & 0.732 \\
\hline 12 & Citronellol & 9.664 & $\mathrm{C}_{10} \mathrm{H}_{20} \mathrm{O}$ & 156.265 & 3.628 \\
\hline 13 & Geraniol & 10.249 & $\mathrm{C}_{10} \mathrm{H}_{18} \mathrm{O}$ & 154.249 & 2.643 \\
\hline 14 & Octanal, 7-hydroxy-3,7-dimethyl- & 10.807 & $\mathrm{C}_{10} \mathrm{H}_{20} \mathrm{O}_{2}$ & 172.265 & 11.728 \\
\hline 15 & 2-Propen-1-ol, 3-phenyl- & 11.055 & $\mathrm{C}_{9} \mathrm{H}_{10} \mathrm{O}$ & 134.175 & 1.465 \\
\hline 16 & $\alpha$-Terpinyl acetate & 11.662 & $\mathrm{C}_{12} \mathrm{H}_{20} \mathrm{O}_{2}$ & 196.286 & 0.740 \\
\hline 17 & 2(3H)-Furanone, dihydro-5-pentyl- & 11.843 & $\mathrm{C}_{9} \mathrm{H}_{16} \mathrm{O}_{2}$ & 156.222 & 0.130 \\
\hline 18 & (E)-3,7-Dimethylocta-2,6-dienyl ethyl carbonate & 12.096 & $\mathrm{C}_{13} \mathrm{H}_{22} \mathrm{O}_{3}$ & 226.312 & 0.153 \\
\hline 19 & Longifolene & 12.543 & $\mathrm{C}_{15} \mathrm{H}_{24}$ & 204.351 & 0.066 \\
\hline 20 & Caryophyllene & 12.72 & $\mathrm{C}_{15} \mathrm{H}_{24}$ & 204.351 & 0.082 \\
\hline 21 & 2-Propenoic acid, 3-phenyl-, ethyl ester & 13.265 & $\mathrm{C}_{11} \mathrm{H}_{12} \mathrm{O}_{2}$ & 176.212 & 0.376 \\
\hline 22 & Lilial & 14.089 & $\mathrm{C}_{14} \mathrm{H}_{20} \mathrm{O}$ & 204.308 & 0.828 \\
\hline 23 & Diethyl Phthalate & 14.922 & $\mathrm{C}_{12} \mathrm{H}_{14} \mathrm{O}_{4}$ & 222.237 & 0.272 \\
\hline 24 & Tricyclo[3.1.0.0(2,4)] hexane,3,3,6,6-tetraethyl-,trans- & 15.732 & $\mathrm{C}_{14} \mathrm{H}_{24}$ & 192.340 & 1.197 \\
\hline 25 & 11,11-Dimethyl-4,8-dimethylenebicyclo[7.2.0]undecan-3-ol & 15.817 & $\mathrm{C}_{15} \mathrm{H}_{24} \mathrm{O}$ & 220.350 & 3.441 \\
\hline 26 & Caryophylla-4(12),8(13)-dien-5 $\alpha$-ol & 15.901 & $\mathrm{C}_{15} \mathrm{H}_{24} \mathrm{O}$ & 220.350 & 0.261 \\
\hline 27 & Phenol, 2-(1,2-dimethyl-2-cyclopenten-1-yl)-, acetate & 16.224 & $\mathrm{C}_{15} \mathrm{H}_{18} \mathrm{O}_{2}$ & 230.302 & 0.092 \\
\hline 28 & Octanal, 2-(phenylmethylene)- & 16.831 & $\mathrm{C}_{15} \mathrm{H}_{20} \mathrm{O}$ & 216.319 & 14.847 \\
\hline 29 & 6-epi-shyobunol & 16.915 & $\mathrm{C}_{15} \mathrm{H}_{26} \mathrm{O}$ & 222.366 & 0.502 \\
\hline 30 & Isolongifolol & 17.208 & $\mathrm{C}_{15} \mathrm{H}_{26} \mathrm{O}$ & 222.366 & 5.826 \\
\hline 31 & $\begin{array}{l}\text { 6-Isopropenyl-4,8a-dimethyl-4a,5,6,7,8,8a-hexahydro-1H naphthalen-2- } \\
\text { one }\end{array}$ & 17.398 & $\mathrm{C}_{15} \mathrm{H}_{22} \mathrm{O}$ & 218.335 & 4.127 \\
\hline 32 & Methyl 3-cis,9-cis,12-cisoctadecatrienoate & 17.451 & $\mathrm{C}_{19} \mathrm{H}_{32} \mathrm{O}_{2}$ & 292.456 & 3.939 \\
\hline 33 & trans-Geranylgeraniol & 17.518 & $\mathrm{C}_{20} \mathrm{H}_{34} \mathrm{O}$ & 290.483 & 1.427 \\
\hline 34 & $\begin{array}{l}\text { 1,1,6-trimethyl-3-methylene-2-(3,6,9,13-tetramethyl-6-ethenye-10,14- } \\
\text { dimethylenepentadec-4-enyl)cyclohexane }\end{array}$ & 17.664 & $\mathrm{C}_{33} \mathrm{H}_{56}$ & 452.798 & 0.432 \\
\hline 35 & Spiro[2.5]octane, 5,5-dimethyl-4-(3-oxobutyl)- & 17.748 & $\mathrm{C}_{14} \mathrm{H}_{24} \mathrm{O}$ & 208.340 & 12.599 \\
\hline 36 & 4-Hexen-1-ol, 6-(2,6,6-trimethyl-1-cyclohexenyl)-4-methyl-, (E)- & 17.907 & $\mathrm{C}_{16} \mathrm{H}_{28} \mathrm{O}$ & 236.393 & 1.343 \\
\hline 37 & 3a,9-Dimethyldodecahydrocyclohepta[d]inden-3-one & 18.067 & $\mathrm{C}_{16} \mathrm{H}_{26} \mathrm{O}$ & 234.377 & 0.590 \\
\hline 38 & 3-Hexen-1-ol, 6-(2,6,6-trimethyl-1-cyclohexenyl)-4-methyl-, (E)- & 18.116 & $\mathrm{C}_{16} \mathrm{H}_{28} \mathrm{O}$ & 236.393 & 0.386 \\
\hline 39 & $\begin{array}{l}\text { 1-Naphthalenepropanol, } \alpha \text {-ethenyldecahydro- } \alpha, 5,5,8 \mathrm{a} \text {-tetramethyl-2- } \\
\text { methylene-, }\left[1 \mathrm{~S}-\left[1 \alpha\left(\mathrm{R}^{*}\right), 4 \mathrm{a} \beta, 8 \mathrm{a} \alpha\right]\right]-\end{array}$ & 18.266 & $\mathrm{C}_{20} \mathrm{H}_{34} \mathrm{O}$ & 290.483 & 0.598 \\
\hline 40 & 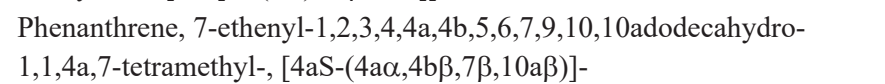 & 18.426 & $\mathrm{C}_{20} \mathrm{H}_{32}$ & 272.468 & 0.357 \\
\hline 41 & Hexadeca-2,6,10,14-tetraen-1-ol, 3,7,11,16-tetramethyl- & 18.665 & $\mathrm{C}_{20} \mathrm{H}_{34} \mathrm{O}$ & 290.483 & 0.239 \\
\hline 42 & (2,6,6-Trimethylcyclohex-1-enylmethanesulfonyl)benzene & 18.785 & $\mathrm{C}_{16} \mathrm{H}_{22} \mathrm{O}_{2} \mathrm{~S}$ & 278.410 & 0.290 \\
\hline 43 & Methyl 10-trans,12-cisoctadecadienoate & 20.366 & $\mathrm{C}_{19} \mathrm{H}_{34} \mathrm{O}_{2}$ & 294.472 & 0.082 \\
\hline 44 & 9,12,15-Octadecatrienoic acid, methyl ester, (Z,Z,Z)- & 20.428 & $\mathrm{C}_{19} \mathrm{H}_{32} \mathrm{O}_{2}$ & 294.472 & 0.142 \\
\hline 45 & 6-(4-Methoxy-phenyl)-2H-[1,3]thiazin & 22.093 & $\mathrm{C}_{11} \mathrm{H}_{11} \mathrm{NOS}$ & 205.276 & 0.333 \\
\hline 46 & Tetramethrin & 24.233 & $\mathrm{C}_{19} \mathrm{H}_{25} \mathrm{NO}_{4}$ & 331.406 & 0.188 \\
\hline
\end{tabular}




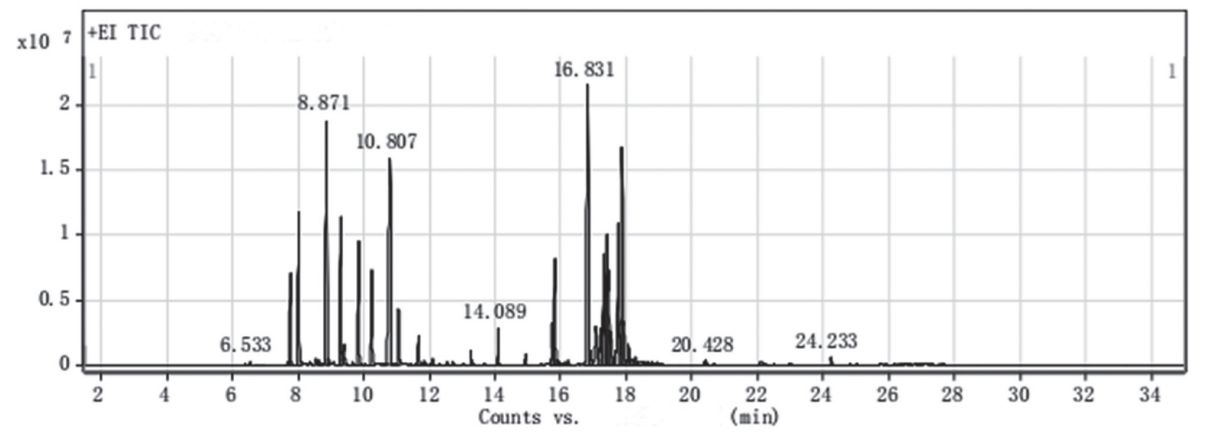

Fig. 1 GC-MS analysis results of PLEO. There were 46 components in the lotus essential oil identified by GC-MS.

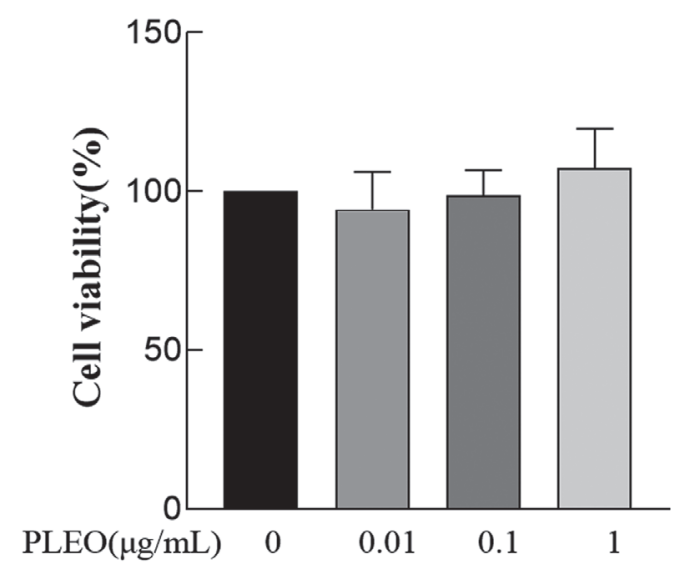

Fig. 2 Effects of PLEO on cell viability in HepG2 cells (A). There was no significant change of cell viability in HeG2 cells after treated with PLEO, even at the highest concentration of $1 \mu \mathrm{g} / \mathrm{mL}$.

0.05 or 0.01 ). The inhibition on inflammation is beneficial for PLEO to intervene the FFA induced steatosis.

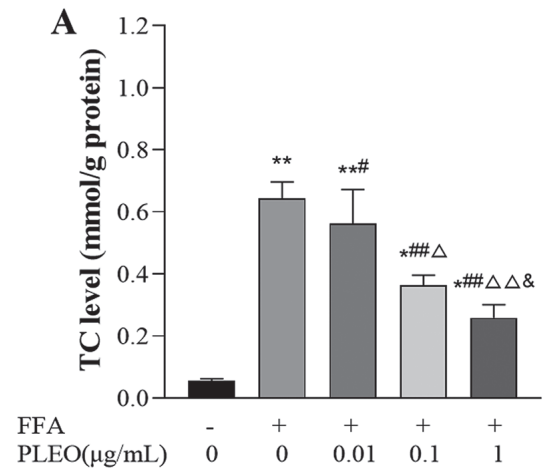

\subsection{Effects of PLEO on expression of PI3K/Akt, NF-kB and lipid metabolism associated enzymes in HepG2 cells}

In order to reveal the possible mechanism of PLEO on FFA induced steatosis, the expression of PI3K/Akt, NF-кB and lipid metabolism associated enzymes was determined in the present study. After treated with FFA, the expression of PI3K, Akt and their phosphorylation significantly decreased (Figs. 6A and 6B, compared with control group, $p<0.05$ or 0.01 ). PI3K/Akt signal pathway is an important one involving in the insulin signaling and is related with the insulin sensitivity. The results revealed that FFA induced insulin resistance in the HepG2 cells via inhibition of PI3K/ Akt signal pathway. PLEO significantly increased the expression of PI3K, Akt and their phosphorylation at a concentration of 0.1 and $1 \mu \mathrm{g} / \mathrm{mL}$, respectively (Figs. $6 \mathrm{~A}$ and $6 \mathrm{~B}$, compared with FFA group, $p<0.05$ or 0.01 ), indicating an improvement of insulin resistance. The expression of

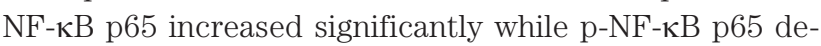
creased in FFA treated HepG2 cells (Figs. 6A and 6C, compared with control group, $p<0.01)$. After treated with PLEO, the expression of NF- $\mathrm{KB}$ p65 decreased while p-NF-кB p65 increased (Figs. 6A and 6C, compared with FFA group, $p<0.05$ or 0.01 ). The inhibition on NF-кB p65

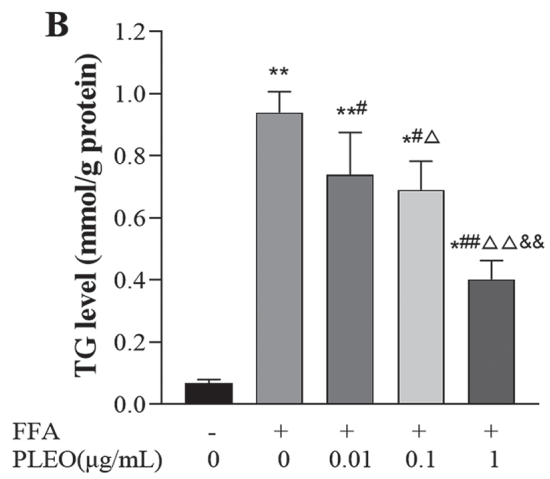

Fig. 3 Effects of PLEO treatment on TC and TG contents in the FFA treated HepG2 cells. PLEO significantly decreased TC (A) and TG (B) contents in the HepG2 cells treated with FFA, showing a dose dependent manner. $* p<0.05, * * p<$ 0.01 versus Control group; ${ }^{\#} p<0.05,{ }^{\# \#} p<0.01$ versus FFA treated group; ${ }^{\triangle} p<0.05,{ }^{\triangle} p<0.01$ versus PLEO $(0.01$ $\mu \mathrm{g} / \mathrm{mL}) ;{ }^{\&} p<0.05,{ }^{\& \&} p<0.01$ versus PLEO $(0.1 \mu \mathrm{g} / \mathrm{mL})$. 

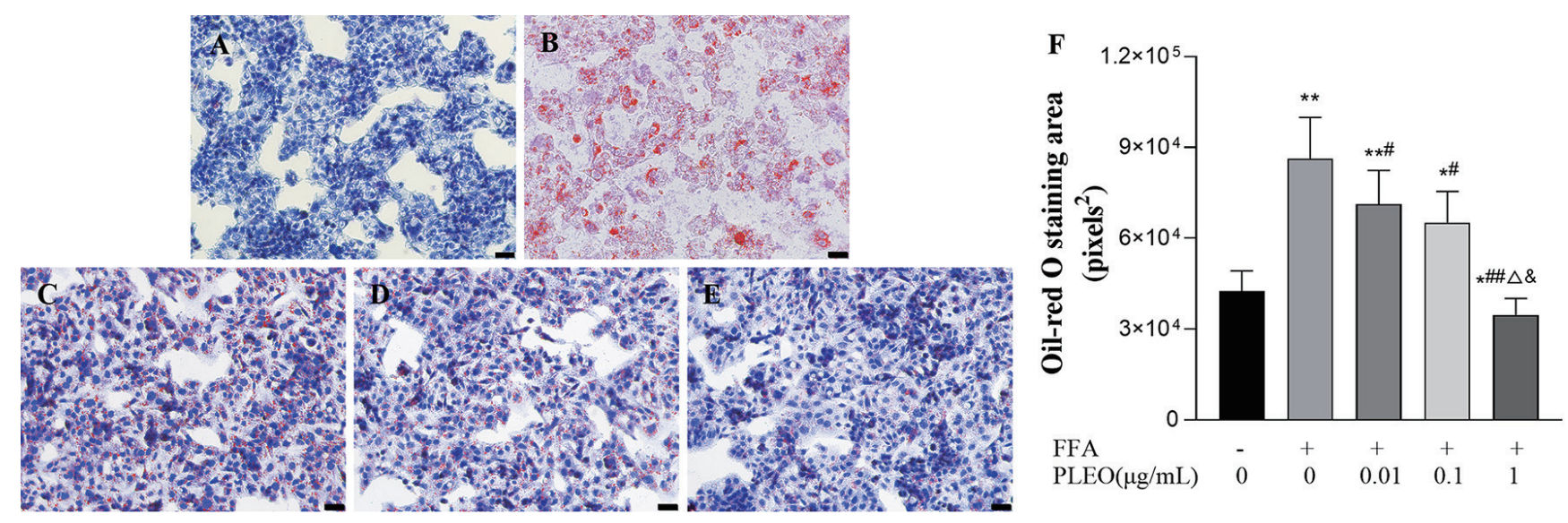

Fig. 4 Oil Red O staining of HepG2 Cells $(\mathrm{A}-\mathrm{E})$ and quantitative analysis $(\mathrm{F})$. When compared with the untreated cells $(\mathrm{A})$, a large amount of lipid accumulated in the hepatic cells (B). After treated with PLEO at a concentration of 0.01(C), $0.1(\mathrm{D})$ and $1 \mu \mathrm{g} / \mathrm{mL}(\mathrm{E})$, lipid accumulation significantly decreased to varying degrees. Red dot shows lipid droplet; pale blue circle shows cell nucleus. ${ }^{*} p<0.05,{ }^{* *} p<0.01$ versus Control group; ${ }^{\#} p<0.05,{ }^{\#} p<0.01$ versus FFA treated group; ${ }^{\wedge} p<0.01$ versus PLEO $(0.01 \mu \mathrm{g} / \mathrm{mL}) ;{ }^{\&} p<0.05$ versus PLEO $(0.1 \mu \mathrm{g} / \mathrm{mL})$. Bars: $20 \mu \mathrm{m}$.
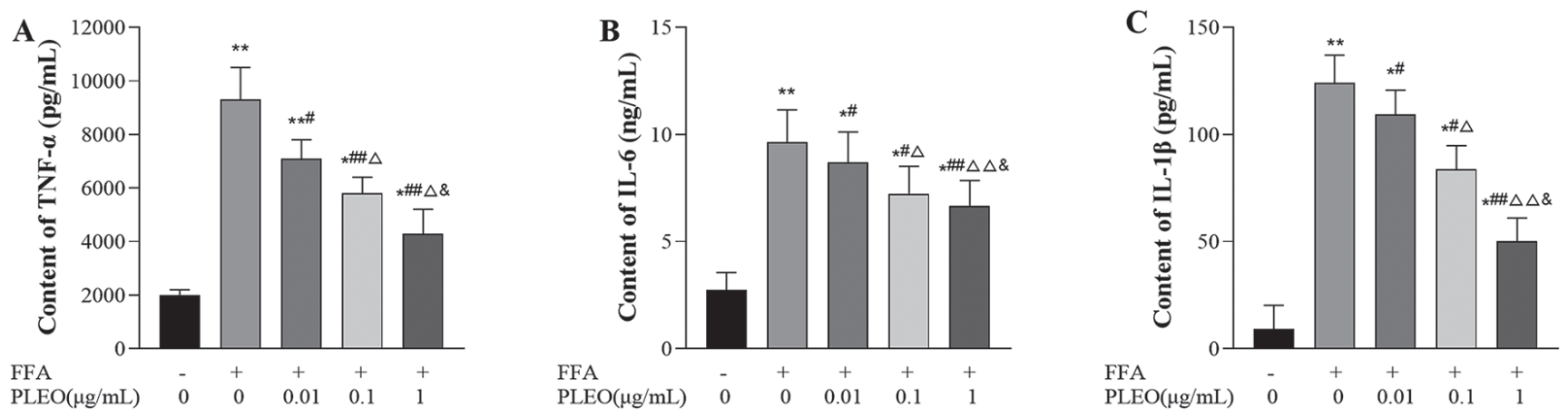

Fig. 5 Effects of PLEO treatment on inflammatory mediators in the FFA treated HepG2 cells. PLEO significantly decreased TNF- $\alpha(\mathrm{A})$, IL-6 (B) and IL-1 $\beta(\mathrm{C})$ contents in the HepG2 cells treated with FFA, showing a dose dependent manner. ${ }^{*} p<0.05,{ }^{* *} p<0.01$ versus Control group; ${ }^{*} p<0.05,{ }^{\# *} p<0.01$ versus FFA treated group; ${ }^{\triangle} p<0.05,{ }^{\Delta}{ }^{\Delta} p<0.01$ versus PLEO $(0.01 \mu \mathrm{g} / \mathrm{mL}) ; \& \mathrm{p}<0.05$ versus PLEO $(0.1 \mu \mathrm{g} / \mathrm{mL})$.

expression and activation on p-NF- $\mathrm{kB}$ p65 of PLEO attribute to the inhibition of inflammation induce by FFA. Furthermore, the expression of FAS, ACC and SCD-1 in FFA treated HepG2 cells increased, while the expression of CPT-1 decreased(Figs. 6A and 6D, compared with the control group, $p<0.05$ or 0.01 ), which might be involved in cellular lipid accumulation. After PLEO treatment, the expression of FAS, ACC and SCD-1 decreased, while the expressions of CPT-1 increased (Figs. 6A and 6D, compared with the FFA group, $p<0.05$ or 0.01 ) which resulted in decreased lipid accumulation.

\section{Discussion}

The liver, the body's largest solid organ, plays a key role in nutrient storage, metabolism, synthesis of new molecules, purification of toxic chemicals and other physiologi- cal processes ${ }^{11)}$. In recent decades, NAFLD has become the most common cause of chronic liver dysfunction. NAFLD represents a wide spectrum of disease stages, extending from simple steatosis (triglycerides accumulation in the liver), nonalcoholic steatohepatitis (steatosis with inflammation) to fibrosis, which may eventually progress to cirrhosis and hepatocellular carcinoma ${ }^{12}$. Therefore, it is urgent to develop new drugs for NAFLD treatment. FFA treated HepG2 cells is an in vitro model of steatosis commonly applied to study the hepatocellular consequences of lipid accumulation. HepG2 cells have the advantages of high availability, simple operation, almost infinite life, stable phenotype and so on ${ }^{13)}$. The functional properties of HepG2 cells have been extensively studied, expressing many different liver functions, such as synthesis and secretion of plasma protein and bile acid, metabolism of cholesterol, triglyceride and lipoprotein, glycogen synthesis or insulin signaling ${ }^{14,15)}$. In the present study, the mixture of 
A

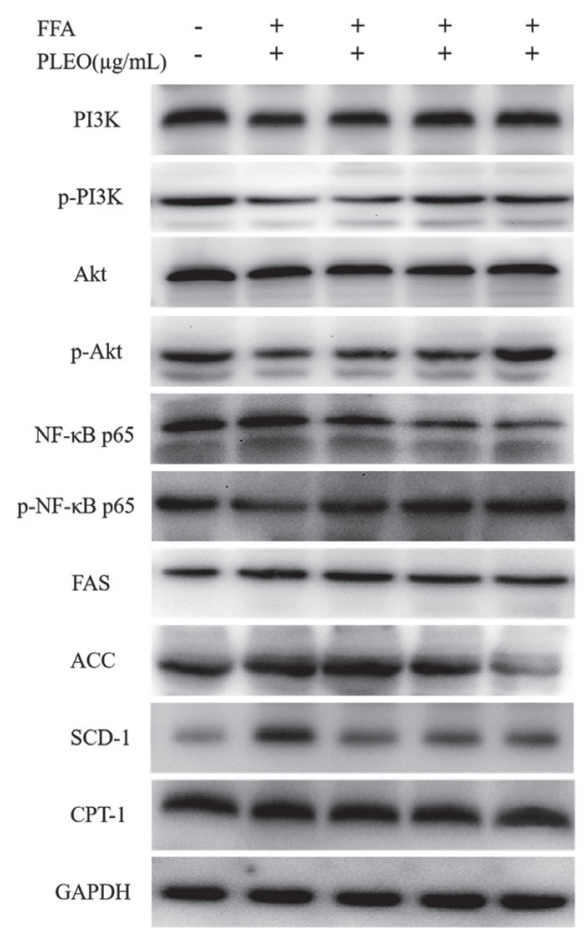

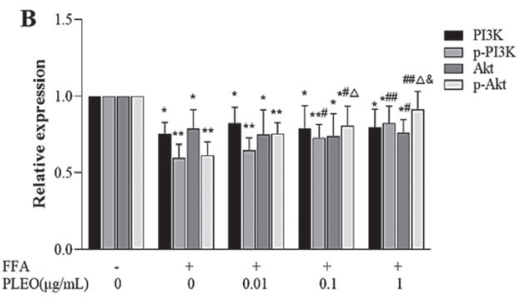
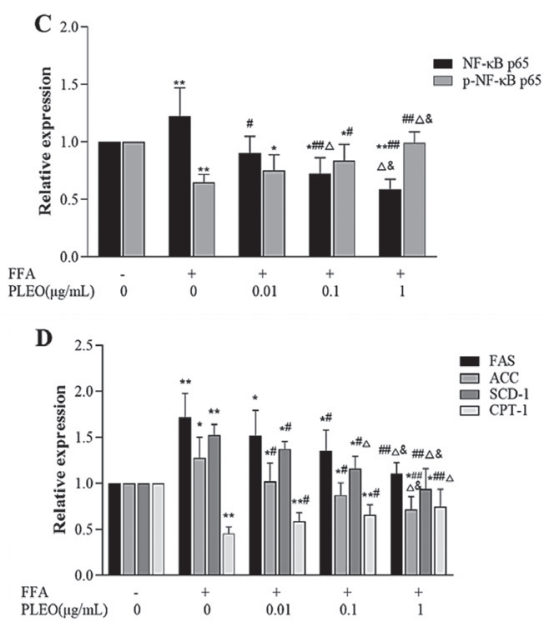

Fig. 6 Effects of PLEO treatment on the protein expressions of PI3K/Akt, NF-кB and lipid metabolism associated enzymes in the FFA treated HepG2 cells. WB bands were shown in (A) and the expression of PI3K/Akt (B), NF-kB(C) and lipid metabolism associated enzymes(D) was quantitively analyzed. There was an inhibition of p-PI3K, p-Akt, p-NF-kB, SCD-1, CPT-1 expressions while an activation of NF-kB expression induced by FFA. After treated with PLEO, the above protein expressions were restored in different degrees. ${ }^{*} p<0.05,{ }^{*} p<0.01$ versus Control group; ${ }^{*} p<0.05$, ${ }^{\#} p<0.01$ versus FFA treated group; ${ }^{\triangleright} p<0.05$ versus PLEO $(0.01 \mu \mathrm{g} / \mathrm{mL})$.

OA and PA (2:1) was employed to induce liver cellular steatosis in the HepG2 cells. As expected, the contents of TC and TG increased significantly after treatment of FFA, indicating a lipid accumulation occurred. Oil red O staining exhibited a typical pathomorphologic changes of steatosis. Furthermore, the contents of TNF- $\alpha$, IL- 6 and IL- $1 \beta$ increased significantly, indicating that there accompanied by inflammation after FFA mixture induced lipid accumulation. All the above changes in the FFA treated HepG2 cells were similar with the key pathological characters of NAFLD in humans, which was an ideal in vitro model of NAFLD. Subsequently, we detected the effect of PLEO on lipid accumulation and inflammation induced by FFA mixture. The results revealed that PLEO significantly decreased TG, TC, TNF- $\alpha$, IL- 6 and IL- $1 \beta$ contents and alleviated cellular steatosis induced by FFA, showing a potential therapeutic effect on NAFLD. There were four groups of ingredients in PLEO recognized by GC-MS, including aliphatic aldehydes, terpenoids, lipids and aromatic hydrocarbons. 2-(phenylmethylene)-Octanal was the most abundant aliphatic compound detected. The identified terpenoids mainly included isolongifolol (5.83\%), L-alphaterpineol (4.56\%), citronellol (3.63\%), geraniol (2.64\%), linalool $(2.45 \%)$, etc. Terpenoids represent the largest group of ingredients consisting of essential oil which account for its pharmaceutical activity ${ }^{16)}$. Alpha-terpineol is a monoterpenoid widely found in essential oil with various bioactive such as antioxidant and anti-inflammatory activity, accounting for $4.56 \%$ in LEO. Recent report suggested that alpha-terpineol improved the insulin sensibility and reduced serum TNF- $\alpha$ and IL-1 $\beta$ levels in rats fed a high-fat diet ${ }^{17)}$. Geraniol is an acyclic isoprenoid monoterpene isolated from LEO and accounts for 2.64\%. Geraniol is potentially applied as a drug based on its various pharmacological properties, including anti-inflammatory, anticancer, antimicrobial, antioxidative, cardioprotective, hepatoprotective and neuroprotective activities ${ }^{18)}$. Linalool, a monoterpene alcohol, accounts for $2.45 \%$ in LEO. Linalool inhibited the production of TNF- $\alpha$ and IL- 6 induce by LPS both in vitro and in vivo ${ }^{19)}$. Furthermore, linalool significantly inhibited lipid production, decreased cholesterol and maintained body weight through multiple mechanisms $^{20,21)}$. Based on these reports, it was speculated that terpenoids in LEO, especially L-alpha-terpineol, geraniol and linalool, were the key potent part contributing to free fatty acid induced steatosis. However, the exact active ingredients and detailed possible mechanisms need for further research. 
The accumulation of a large amount of fat in the liver leads to steatosis of liver parenchymal cells, which is an important inducer of NAFLD. FAS, ACC, and SCD-1 are three important enzymes participate in fatty acid synthesis. ACC catalyzes malonyl-CoA, an intermediate metabolite of lipid formation and oxidation ${ }^{22)}$. FAS is a rate-limiting enzyme to catalyze the final step of lipid synthesis in the liver $^{23)}$. SCD-1 is another key enzyme involving in lipid synthesis, which catalyzes fatty acid elongation and desaturation $^{24)}$. At present, there are two signal pathways involving in insulin signaling that up-regulate the expression of these enzymes. One is the sterol regulated element-binding protein-1C (SREBP-1C), and the other is carbohydrate responsive element binding protein $(\mathrm{ChREBP})^{25)}$. CPT-1 is the rate-limiting enzyme of fatty acid oxidation. In the process of fatty acid oxidation, CPT-1 is a key regulatory site for fatty acid flow into mitochondria, and abnormal fatty acid oxidation process is also an important pathogenic factor of NAFLD. CPT- 1 controls $\beta$-oxidation of fatty acids ${ }^{26)}$. In our present study, the expression of FAS, ACC and SCD-1 in the HepG2 cells treated with FFA increased significantly, while the expression of CPT-1 decreased when compared with the control group. These changes resulted in lipid accumulation in the cells. After PLEO was applied, the expression of FAS, ACC and SCD-1 decreased, while the expression of CPT-1 increased, indicating that PLEO inhibited fatty acid synthesis and promoted its oxidation. The regulation of PLEO on the lipid metabolism associated enzymes might be involved in the therapeutic effect of PLEO on steatosis induced by FFA.

The PI3K/Akt signaling pathway is involved in the regulation of various cell functions, such as proliferation, apoptosis and autophagy. PI3Ks are members of the intracellular lipid kinase family that phosphorylate the hydroxyl groups of 3'-phosphatidylinositol and phosphoinositides ${ }^{27)}$. Akt is a key downstream target of PI3K and a central mediator of the PI3K pathway. It plays a variety of downstream effects through phosphorylation of many substrates involved in cell survival, proliferation, metabolism and movement. Furthermore, PI3K/Akt is an important pathway involving in insulin signaling. Insulin activates PI3K and downstream enzymes via its receptor to regulate glucose and lipid metabolism ${ }^{28)}$. Damage of PI3K/AKT results in insulin resistance, which increases de novo lipogenesis and promotes FFA flux to the liver, therefore leads to metabolic disorders such as NAFLD, obesity and type 2 diabetes $^{29,30)}$. Cumulative evidences suggest that the PI3K/Akt signaling pathway plays a role in lipid metabolism and liver injury which is related with insulin resistance ${ }^{31)}$. The activation of PI3K/Akt can inhibit fat generation, delay excessive fat deposition, and reduce liver steatosis ${ }^{32)}$. Phosphorylation of Akt up-regulates lipase production and promotes lipid metabolism in hepatocytes ${ }^{33)}$. Therefore, PI3K/Akt has been an important target for ameliorating insulin resistance in the treatment of $\mathrm{NAFLD}^{34-36)}$. Here we confirmed that there was a significant decrease in expression of Akt, PI3K and their phosphorylation levels in the FFA treated HepG2 cells. Moreover, PLEO significantly increased the expression of $\mathrm{p}$-Akt and $\mathrm{p}-\mathrm{PI} 3 \mathrm{~K}^{37,38)}$, which resulted in alleviation of liver cellular steatosis through improving insulin resistance and was a new strategy for NAFLD treatment.

$\mathrm{NF}-\kappa \mathrm{B}$ is a major transcriptional regulator of inflammation and cell death in the development of chronic liver disease $^{39)}$. It is always activated in NAFLD in response to inflammation and regulates the functions of hepatocytes, Kupffer cells, and hepatic stellate cells by inducing the expression of inflammation-related genes, essentially promoting inflammation of the liver during the progression of $\mathrm{NAFLD}^{40)}$. Our Western blotting analysis data showed that expression of NF- $\mathrm{B}$ p 65 increased while its phosphorylation decreased in the HepG2 cells treated with FFA, indicating that NF- $\mathrm{BB}$ p65 activation mediated the inflammatory injury in the cellular model of NAFLD. After treated with PLEO, p-NF- $\mathrm{B}$ p65 expression increased significantly while NF-кB p65 expression decreased, indicating an inhibition of inflammatory injury induced by FFA.

\section{Conclusion}

In conclusion, PLEO is a complex essential oil extracted from pink lotus flowers. It decreased TC and TG contents and inhibited lipid accumulation in the FFA treated HepG2 cells, exhibiting a potential therapeutic effect on NAFLD. The underlying mechanism might be involved in its regulation on the lipid metabolism associated enzymes, inhibition of NF-кB signals and inflammatory mediators release, activation of PI3K/Akt signal to improve insulin resistance. PLEO is a promising natural plant essential oil deserving for further research and development.

\section{Acknowledgments}

We thank Healzone Co. Ltd (Qingdao, China) for providing essential oil and experimental conditions.

\section{Contributions}

Runzhou Sun: performed research and wrote the manuscript. Ruixin Xiao: performed research. Pengfei Lv: performed research. Feifei Guo: analyzed data. Yanling Gong: designed research. Meixing Yan: provided financial support. 


\section{Conflict of Interest}

None of the authors has personal or financial conflicts of interest.

\section{References}

1) Abood, S.; Veisaga, M.L.; López, L.A.; Barbieri, M.A. Dehydroleucodine inhibits mitotic clonal expansion during adipogenesis through cell cycle arrest. Phytother. Res. 32, 1583-1592(2018).

2) Buzzetti, E.; Pinzani, M.; Tsochatzis, E.A. The multiple-hit pathogenesis of non-alcoholic fatty liver disease (NAFLD). Metabolism 65, 1038-1048(2016).

3) Donato, M.T.; Tolosa, L.; Gómez-Lechón, M.J. Culture and functional characterization of human hepatoma HepG2 cells. Methods Mol. Biol. 1250, 77-93 (2015).

4) Dongiovanni, P.; Valenti, L.; Ludovica Fracanzani, A.; Gatti, S.; Cairo, G.; Fargion, S. Iron depletion by deferoxamine up-regulates glucose uptake and insulin signaling in hepatoma cells and in rat liver. Am. J. Pathol. 172, 738-747 (2008).

5) Gómez-Lechón, M.J.; Donato, M.T.; Martínez-Romero, A.; Jiménez, N.; Castell, J.V.; O’Connor, J.E. A human hepatocellular in vitro model to investigate steatosis. Chem. Biol. Interact. 165, 106-116(2007).

6) Hein, G.J.; Bernasconi, A.M.; Montanaro, M.A.; PellonMaison, M.; Finarelli, G. et al. Nuclear receptors and hepatic lipidogenic enzyme response to a dyslipidemic sucrose-rich diet and its reversal by fish oil n-3 polyunsaturated fatty acids. Am. J. Physiol. Endocrinol. Metab. 298, E429-E439(2010).

7) Huang, X.; Liu, G.; Guo, J.; Su, Z. The PI3K/AKT pathway in obesity and type 2 diabetes. Int. J. Biol. Sci. 14, 1483-1496 (2018).

8) Li, J.; Chi, Y.; Wang, C.; Wu, J.; Yang, H. et al. Pancreatic-derived factor promotes lipogenesis in the mouse liver: Role of the Forkhead box 1 signaling pathway. Hepatology 53, 1906-1916 (2011).

9) Liao, X.; Song, L.; Zhang, L.; Wang, H.; Tong, Q. et al. LAMP3 regulates hepatic lipid metabolism through activating PI3K/Akt pathway. Mol. Cell. Endocrinol. 470, 160-167 (2018).

10) Liu, T.; Tan, F.; Long, X.; Pan, Y.; Mu, J. et al. Improvement effect of lotus leaf flavonoids on carbon tetrachloride-induced liver injury in mice. Biomedicines 8 (2), 41 (2020).

11) Luedde, T.; Schwabe, R.F. NF-кB in the liver--linking injury, fibrosis and hepatocellular carcinoma. Nat. Rev. Dis. Primers 8, 108-118(2011).

12) Meier, M.; Klein, H.H.; Kramer, J.; Drenckhan, M.; Schütt, M. Calpain inhibition impairs glycogen syntheses in HepG2 hepatoma cells without altering insulin signaling. J. Endocrinol. 193, 45-51 (2007).
13) Morgan, K.; Uyuni, A.; Nandgiri, G.; Mao, L.; Castaneda, L. et al. Altered expression of transcription factors and genes regulating lipogenesis in liver and adipose tissue of mice with high fat diet-induced obesity and nonalcoholic fatty liver disease. Eur. J. Gastroenterol. Hepatol. 20, 843-854(2008).

14) Pappachan, J.M.; Babu, S.; Krishnan, B.; Ravindran, N.C. Non-alcoholic fatty liver disease: A clinical update. J. Clin. Transl. Hepatol. 5, 384-393 (2017).

15) Paudel, K.R.; Panth, N. Phytochemical profile and biological activity of Nelumbo nucifera. Evid. Based Complement Alternat. Med. 789124(2015).

16) de Groot, A.C.; Schmidt, E. Essential oils, Part III: Chemical composition. Dermatitis 27, 161-169 (2016).

17) Sousa, G.M.; Cazarin, C.; Maróstica Junior, M.R.; Lamas, C.A.; Quitete, V.; Pastore, G.M.; Bicas, J.L. The effect of $\alpha$-terpineol enantiomers on biomarkers of rats fed a high-fat diet. Heliyon. 6, e03752 (2020).

18) Lei, Y.; Fu, P.; Jun, X.; Cheng, P. Pharmacological properties of geraniol - A review. Planta Med. 85, 48-55 (2019).

19) Huo, M.; Cui, X.; Xue, J.; Chi, G.; Gao, R. et al. Anti-inflammatory effects of linalool in RAW 264.7 macrophages and lipopolysaccharide-induced lung injury model. J. Surg. Res. 180, e47-e54(2013).

20) Cheng, B.H.; Sheen, L.Y.; Chang, S.T. Hypolipidemic effects of S- (+)-linalool and essential oil from Cinnamomum osmophloeum ct. linalool leaves in mice. $J$. Tradit. Complement Med. 8, 46-52(2017).

21) Cho, S.Y.; Jun, H.J.; Lee, J.H.; Jia, Y.; Kim, K.H.; Lee, S.J. Linalool reduces the expression of 3-hydroxy-3-methylglutaryl CoA reductase via sterol regulatory element binding protein-2- and ubiquitin-dependent mechanisms. FEBS Lett. 585, 3289-3296. (2011).

22) Postic, C.; Girard, J. Contribution of de novo fatty acid synthesis to hepatic steatosis and insulin resistance: lessons from genetically engineered mice. J. Clin. Invest. 118, 829-838(2008).

23) Shen, Y.; Guan, Y.; Song, X.; He, J.; Xie, Z. et al. Polyphenols extract from lotus seedpod (Nelumbo nucifera Gaertn. ): Phenolic compositions, antioxidant, and antiproliferative activities. Food Sci. Nutr. 7, 3062-3070 (2019).

24) Sheikh, S.A. Ethno-medicinal uses and pharmacological activities of lotus (Nelumbo nucifera). J. Med. Plants Stud. 2(6), 42-46(2014).

25) Sumida, Y.; Yoneda, M. Current and future pharmacological therapies for NAFLD/NASH. J. Gastroenterol. 53, 362-376 (2018).

26) Sumida, Y.; Nakashima, T.; Yoh, T.; Furutani, M.; Hirohama, A. et al. Serum thioredoxin levels as a predictor of steatohepatitis in patients with nonalcoholic fatty liver disease. J. Hepatol. 38, 32-38 (2003).

27) Takahashi, Y.; Sugimoto, K.; Inui, H.; Fukusato, T. Cur- 
rent pharmacological therapies for nonalcoholic fatty liver disease/nonalcoholic steatohepatitis. World J. Gastroenterol. 21, 3777-3785(2015).

28) Kanai, F.; Ito, K.; Todaka, M.; Hayashi, H.; Kamohara, S. et al. Insulin-stimulated GLUT4 translocation is relevant to the phosphorylation of IRS-1 and the activity of PI3-kinase. Biochem. Biophys. Res. Commun. 195, 762-768 (1993).

29) Maurice, J.; Manousou, P. Non-alcoholic fatty liver disease. Clin. Med. (Lond.) 18, 245-250 (2018).

30) Wang, H.; Liu, Y.; Wang, D.; Xu, Y.; Dong, R. et al. The upstream pathway of mTOR-mediated autophagy in liver diseases. Cells 8, 1597 (2019).

31) Tilg, H.; Diehl, A.M. Cytokines in alcoholic and nonalcoholic steatohepatitis. N. Engl. J. Med. 343, 14671476 (2000).

32) Trefts, E.; Gannon, M.; Wasserman, D.H. The liver. Curr. Biol. 27, R1147-R1151 (2017).

33) Tseng, H.C.; Tsai, P.M.; Chou, Y.H.; Lee, Y.C.; Lin, H.H.; Chen, J.H. In vitro and in vivo protective effects of flavonoid-enriched lotus seedpod extract on lipopolysaccharide-induced hepatic inflammation. Am. J. Chin. Med. 47, 153-176(2019).

34) Zhang, Y.; Hai, J.; Cao, M.; Zhang, Y.; Pei, S.; Wang, J.; Zhang, Q. Silibinin ameliorates steatosis and insulin resistance during non-alcoholic fatty liver disease development partly through targeting IRS-1/PI3K/Akt pathway. Int. Immunopharmacol. 17, 714-720 (2013).

35) Xu, H.; Zhou, Y.; Liu, Y.; Ping, J.; Shou, Q. et al. Metformin improves hepatic IRS2/PI3K/Akt signaling in insulin-resistant rats of NASH and cirrhosis. J. Endocrinol. 229, 133-144(2016).

36) Zheng, X.; Zhao, M.G.; Jiang, C.H.; Sheng, X.P.; Yang, H.M. et al. Triterpenic acids-enriched fraction from Cyclocarya paliurus attenuates insulin resistance and hepatic steatosis via PI3K/Akt/GSK3 $\beta$ pathway. Phytomedicine 66, 153130 (2020).

37) Chen, S.H.; Liu, X.N.; Peng, Y. MicroRNA-351 eases insulin resistance and liver gluconeogenesis via the PI3K/AKT pathway by inhibiting FLOT2 in mice of gestational diabetes mellitus. J. Cell Mol. Med. 23, 5895-5906 (2019).

38) Yan, J.; Wang, C.; Jin, Y.; Meng, Q.; Liu, Q. et al. Catalpol ameliorates hepatic insulin resistance in type 2 diabetes through acting on AMPK/NOX4/PI3K/AKT pathway. Pharmacol. Res. 130, 466-480 (2018).

39) Wang, H.; Liu, Y.; Wang, D.; Xu, Y.; Dong, R. et al. The upstream pathway of mTOR-mediated autophagy in liver diseases. Cells 8, 1597 (2019).

40) Zang, Y.; Wang, T.; Xie, W.; Wang-Fischer, Y.L.; Getty, L. et al. Regulation of acetyl CoA carboxylase and carnitine palmitoyl transferase-1 in rat adipocytes. Obes. Res. 13, 1530-1539(2005).

CC BY 4.0 (Attribution 4.0 International). This license allows users to share and adapt an article, even commercially, as long as appropriate credit is given. That is, this license lets others copy, distribute, remix, and build upon the Article, even commercially, provided the original source and Authors are credited. 